\title{
DISAPPEARANCE OF THE PROGENITOR OF SUPERNOVA iPTF13bvn
}

\author{
Gastón Folatelli ${ }^{1,2}$, Schuyler D. Van Dyk ${ }^{3}$, Hanindyo Kuncarayakti ${ }^{4,5}$, Keilchi Maeda ${ }^{2,6}$, Melina C. Bersten ${ }^{1,2}$, \\ Ken’ichi Nomoto $^{2,15}$, Giuliano Pignata, ${ }^{4,7}$, Mario Hamuy ${ }^{4,5}$, Robert M. Quimby ${ }^{2,8}$, WeiKang Zheng ${ }^{9}$, \\ Alexei V. Filippenko ${ }^{9}$, Kelsey I. Clubb ${ }^{9}$, Nathan Smith ${ }^{10}$, Nancy Elias-Rosa ${ }^{11}$, Ryan J. Foley ${ }^{12,13}$, and \\ AdAM A. Miller ${ }^{14,16}$ \\ ${ }^{1}$ Facultad de Ciencias Astronómicas y Geofísicas, Universidad Nacional de La Plata, Instituto de Astrofísica de La Plata (IALP), \\ CONICET, Paseo del Bosque S/N, B1900FWA La Plata, Argentina; gaston.folatelli@ipmu.jp \\ ${ }^{2}$ Kavli Institute for the Physics and Mathematics of the Universe (WPI), The University of Tokyo, Kashiwa, Chiba 277-8583, Japan \\ ${ }_{4}^{3}$ IPAC/Caltech, Mailcode 100-22, Pasadena, CA 91125, USA \\ ${ }^{4}$ Millennium Institute of Astrophysics (MAS), Santiago, Chile \\ ${ }^{5}$ Departamento de Astronomía, Universidad de Chile, Casilla 36-D, Santiago, Chile \\ ${ }^{6}$ Department of Astronomy, Kyoto University, Kitashirakawa-Oiwake-cho, Sakyo-ku, Kyoto 606-8502, Japan \\ Departamento de Ciencias Fisicas, Universidad Andres Bello, Avda. Republica 252, Santiago, Chile \\ 8 Department of Astronomy, San Diego State University, 5500 Campanile Drive, San Diego, CA 92182-1221, USA \\ ${ }^{9}$ Department of Astronomy, University of California, Berkeley, CA 94720-3411, USA \\ ${ }^{10}$ Steward Observatory, University of Arizona, 933 N. Cherry Avenue, Tucson, AZ 85721, USA \\ ${ }^{11}$ INAF-Osservatorio Astronomico di Padova, Vicolo dell'Osservatorio 5, I-35122 Padova, Italy \\ ${ }^{12}$ Astronomy Department, University of Illinois at Urbana-Champaign, 1002 W. Green Street, Urbana, IL 61801, USA \\ ${ }^{13}$ Department of Physics, University of Illinois at Urbana-Champaign, 1110 W. Green Street, Urbana, IL 61801, USA \\ ${ }^{14}$ Jet Propulsion Laboratory, 4800 Oak Grove Drive, MS 169-506, Pasadena, CA 91109, USA \\ Received 2016 April 22; revised 2016 May 25; accepted 2016 June 2; published 2016 July 5
}

\begin{abstract}
Supernova (SN) iPTF13bvn in NGC 5806 was the first Type Ib SN to have been tentatively associated with a progenitor in pre-explosion images. We performed deep ultraviolet (UV) and optical Hubble Space Telescope observations of the SN site 740 days after explosion. We detect an object in the optical bands that is fainter than the pre-explosion object. This dimming is likely not produced by dust absorption in the ejecta; thus, our finding confirms the connection of the progenitor candidate with the SN. The object in our data is likely dominated by the fading SN, implying that the pre-SN flux is mostly due to the progenitor. We compare our revised pre-SN photometry with previously proposed models. Although binary progenitors are favored, models need to be refined. In particular, to comply with our deep UV detection limit, any companion star must be less luminous than a late-O star or substantially obscured by newly formed dust. A definitive progenitor characterization will require further observations to disentangle the contribution of a much fainter SN and its environment.
\end{abstract}

Key words: galaxies: individual (NGC 5806) - stars: evolution - supernovae: general - supernovae: individual (iPTF13bvn)

Supporting material: machine-readable table

\section{INTRODUCTION}

The stellar origin of hydrogen-free core-collapse supernovae $(\mathrm{SNe})$ remains unknown chiefly owing to the lack of detections of progenitor stars in pre-explosion images (Eldridge et al. 2013). The only firm progenitor candidate found thus far is that of iPTF13bvn, a Type Ib supernova (SN Ib) in the galaxy NGC 5806 (Cao et al. 2013). If confirmed, this case can provide important clues about the mechanisms of envelope removal among massive stars. One proposed mechanism for very massive stars $\left(M_{\text {ZAMS }}>25 M_{\odot}\right)$ is strong stellar winds leading to Wolf-Rayet (WR) progenitors (Heger et al. 2003), but such massive progenitors are difficult to reconcile with the large fraction of stripped-envelope explosions (Smith et al. 2011) and their low ejecta masses (Dessart et al. 2011; Drout et al. 2011; Hachinger et al. 2012). The more common alternative is mass transfer in close binary systems (Shigeyama et al. 1990; Podsiadlowski et al. 1992), allowing less-massive stars to lose their envelopes (e.g., Benvenuto et al. 2013). The relative incidence of each type of progenitor is still unknown.

\footnotetext{
${ }^{15}$ Hamamatsu Professor.

${ }^{16}$ Hubble Fellow.
}

Cao et al. (2013) used pre-explosion Hubble Space Telescope (HST) imaging, together with early-time SN observations, to suggest that the progenitor of iPTF13bvn was a compact WR star. Groh et al. (2013b) found that the preexplosion photometry could be fit with models of single WR stars in the initial mass range of $31-35 M_{\odot}$. However, from hydrodynamical light-curve modeling, Bersten et al. (2014) inferred a low pre-SN mass $\left(\sim 3.5 M_{\odot}\right)$ and disfavored a massive WR star; instead, they presented close binary system models that could explain the pre-explosion photometry and the progenitor mass and radius derived from the $\mathrm{SN}$ observations. By analyzing the light curves, Fremling et al. (2014) and Srivastav et al. (2014) also disfavored a massive WR progenitor. Kuncarayakti et al. (2015) argued for a lowmass progenitor based on the strength of oxygen and calcium lines in the late-time spectrum. Subsequently, Eldridge et al. (2015, hereafter E15) revised the pre-explosion photometry and found the progenitor to be brighter than measured by $\mathrm{Cao}$ et al. (2013; see also our measurements in Section 2). With the new magnitudes and using binary evolution calculations, E15 argued in favor of the binary scenario. The same conclusion was found by Kim et al. (2015). Assuming the binary configurations discussed by Bersten et al. (2014), Hirai \& 
Table 1

Summary of $H S T$ Observations

\begin{tabular}{|c|c|c|c|c|c|}
\hline UT Date & $\begin{array}{l}\text { Instrument/ } \\
\text { Detector }\end{array}$ & Filter & $\begin{array}{l}\text { Exposure } \\
\text { (s) }\end{array}$ & $\begin{array}{l}\text { Program } \\
\text { ID }\end{array}$ & $\begin{array}{l}\text { Program } \\
\text { PI }\end{array}$ \\
\hline \multicolumn{6}{|c|}{ Pre-explosion } \\
\hline 2005 Mar 10 & ACS/WFC & F555W & 1400 & GO-10187 & Smartt \\
\hline 2005 Mar 10 & ACS/WFC & F814W & 1700 & GO-10187 & Smartt \\
\hline 2015 Jun 26 & WFC3/UVIS & F438W & 5720 & GO-13684 & Van Dyk \\
\hline 2015 Jun 26 & WFC3/UVIS & F555W & 5610 & GO-13684 & Van Dyk \\
\hline 2015 Jun 30 & WFC3/UVIS & $\mathrm{F} 225 \mathrm{~W}$ & 8865 & GO-13822 & Folatelli \\
\hline 2015 Jun 30 & ACS/WFC & F814W & 2345 & GO-13822 & Folatelli \\
\hline
\end{tabular}

Yamada (2015) simulated the effect of the SN shock on the companion star. They found that the companion could bloat and thus evolve to a red-supergiant structure on a timescale of a few years after explosion. We note, however, that similar shock simulations performed by Liu et al. (2015), although for different companion masses, do not predict such a postexplosion evolution.

To better determine the progenitor's nature, the SN site had to be reobserved after the ejecta faded enough to probe the disappearance of the pre-SN object. In this work, we present new $H S T$ observations that reveal a decrease in flux relative to the pre-SN observations, confirming that this is the first $\mathrm{SN} \mathrm{Ib}$ with a progenitor detection. ${ }^{17}$ In Section 2 , we describe the observations and photometry methods. The new data are analyzed in Section 3 to constrain the progenitor nature. We present our conclusions in Section 4.

\section{OBSERVATIONS AND PHOTOMETRY}

We obtained deep imaging of the field of iPTF13bvn 740 days after explosion using HST through Cycle 22 programs GO-13684 and GO-13822. Program GO-13684 was executed between 2015 June 26.37 and 26.60 (UT dates are used herein) with the Wide Field Camera 3 (WFC3) UVIS channel; see Table 1. Program GO-13822 comprised observations obtained on 2015 June 30.63 with WFC3/UVIS (F225W filter) and on June 30.90 UT with the Advanced Camera for Surveys (ACS; F814W filter). The 2015 images are shown in Figure 1, along with the pre-explosion images obtained in 2005 through program GO-10187 with ACS.

The SN location in the pre- and post-explosion images was found by aligning them relative to a F555W image obtained through program GO-12888 with WFC3/UVIS on 2013 September 2.37 when the SN was still very bright. The registration was done with the AstroDrizzle package employing 20 point sources in common. The resulting precision (rms) was 0.081 pixels ( 32 mas) and 0.090 pixels (36 mas) in the $X$ and $Y$ axes, respectively. The F435W, F555W, and F814W images from 2015 show an object at the SN location, whereas the F225W image exhibits no identifiable source at the same place.

After correcting for charge-transfer-efficiency losses and masking of cosmic-ray hits, we performed photometry on the

\footnotetext{
${ }^{17}$ In the final stages of preparing this manuscript, Eldridge \& Maund (2016) independently reported the disappearance of the progenitor candidate using some of the data presented herein.
}

pre- and post-explosion images using the DOLPHOT v2.0 package (Dolphin 2000). Our measurements are listed in Table 2 along with others from the literature. The pre-explosion photometry given here is roughly consistent with that presented by E15 (both their values from DOLPHOT, and the average with DAOPHOT measurements), and it is brighter by $0.3-0.5$ mag than what Cao et al. (2013) measured. In a recent work, Eldridge \& Maund (2016) published photometry of the 2015 F438W and F555W images. Their values are brighter than ours, more significantly so (by $\sim 5 \sigma$ ) in the F555W band. We have tried to reproduce their results by changing the parameters in DOLPHOT, but could not obtain exactly the same values. This may in part be due to the fact that they apply DOLPHOT on the _crj files while we use the _flc files. Additionally, E15 mention that they adopt the recommended DOLPHOT parameters. Among these, the sky-fitting algorithm parameter, FitSky, is suggested to be set as 1 for general purposes in the DOLPHOT v2.0 ACS module manual. However, for a crowded field such as that of iPTF13bvn, the recommendation is to perform the sky fit inside a relatively large photometry aperture by adopting FitSky $=3$ (Dalcanton et al. 2009), which was our choice. If we instead use FitSky $=1$, we obtain $\mathrm{F} 555 \mathrm{~W}=26.37 \pm 0.05 \mathrm{mag}$, in close agreement with Eldridge \& Maund (2016). We also obtain large negative sharpness values, as mentioned by those authors, which is not seen with FitSky $=3$. We conclude that the main source of discrepancy is likely the choice of the sky-fitting algorithm, and that for the field of iPTF13bvn, our procedure is more accurate. Nevertheless, we note that the main conclusions in the present work would not change if we adopted the photometry in E15 and Eldridge \& Maund (2016).

Figure 2 (left panel) shows the $H S T$ magnitudes converted to $B V I$. The conversion was calculated using synthetic photometry from the 306 day spectrum of Kuncarayakti et al. (2015), which gave $B-\mathrm{F} 438 \mathrm{~W}=0.06 \mathrm{mag}, V-\mathrm{F} 555 \mathrm{~W}=-0.03 \mathrm{mag}$, and $I-\mathrm{F} 814 \mathrm{~W}=-0.05 \mathrm{mag}$.

We used the $\mathrm{F} 225 \mathrm{~W}$ image to compute a detection limit at the SN location, employing DOLPHOT to add 50,000 artificial stars near the SN site with a uniform distribution of brightness over 25.4-27.4 mag. DOLPHOT was run on the resulting images to recover the artificial sources. A detection limit of $26.4 \mathrm{mag}$ was found where the recovery fraction dropped to $50 \%$, which is roughly equivalent to a $5 \sigma$ detection limit (Harris 1990). 

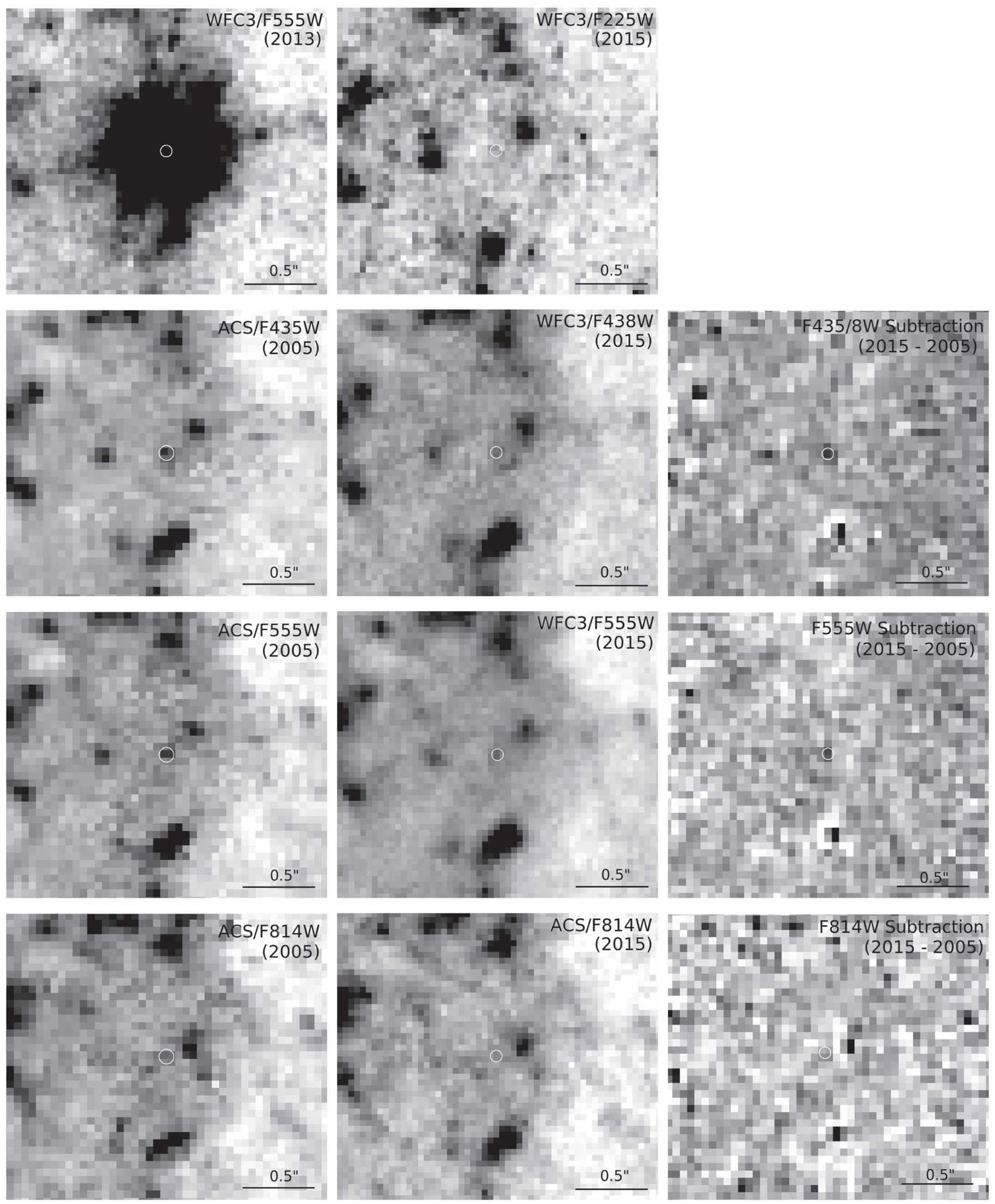

Figure 1. HST images of the site of iPTF13bvn at different epochs. Top left: an image near maximum light to locate the SN. Rest of left column: pre-SN images obtained in 2005. Middle column: new images obtained in 2015. Right column: pre-SN minus post-SN images. The SN location is shown with a white circle of $3 \sigma$ radius. The image scale is indicated. North is up, and east to the left. 
Table 2

Photometry from Pre- and Post-explosion HST Images

\begin{tabular}{lccccl}
\hline \hline UT Date & F225W & F435W/F438W & F555W & F814W & Source \\
\hline 2005 Mar 10 & $\ldots$ & $26.50(15)$ & $26.40(15)$ & $26.10(20)$ & Cao et al. (2013) \\
2005 Mar 10 & $\ldots$ & $25.81(06)$ & $25.86(08)$ & $25.77(10)$ & E15 (DOLPHOT) \\
2005 Mar 10 & $\ldots$ & $25.80(12)$ & $25.80(11)$ & $25.88(24)$ & E15 (Average) \\
2005 Mar 10 & $\ldots$ & $25.99(14)$ & $26.06(13)$ & $25.82(12)$ & This work \\
2015 Jun 26 & $\ldots$ & $26.48(08)$ & $26.33(05)$ & $\ldots$ & Eldridge \& Maund (2016) \\
2015 Jun 26/30 & $>26.4^{\text {a }}$ & $26.62(14)$ & $26.72(08)$ & $26.03(15)$ & This work \\
Subtraction $(2005-2015)$ & $\ldots$ & $27.13(12)$ & $27.12(21)$ & $>27.5^{\text {a }}$ & This work \\
\hline
\end{tabular}

Note. Uncertainties in parentheses in units of $0.01 \mathrm{mag}$.

${ }^{a}$ Limiting magnitude at $50 \%$ detection probability.
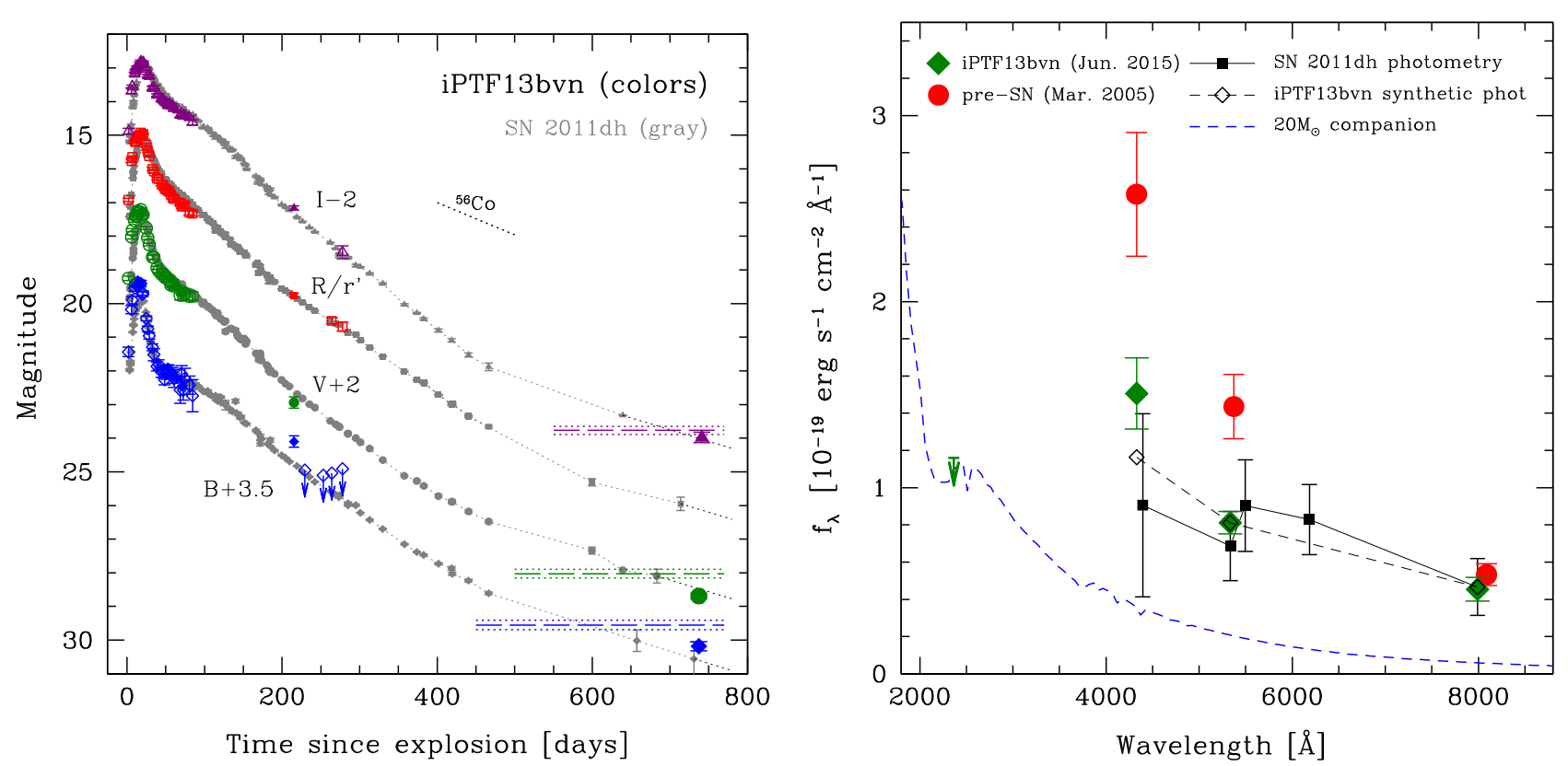

Figure 2. Left panel: BVRI light curves of iPTF13bvn (colored symbols) obtained with KAIT (open), Nickel (small filled), and HST (large filled; converted to BVI). Detection limits in $B$ are indicated with arrows. Gray points connected with dotted lines show the BVRr'I photometry of SN 2011dh from Ergon et al. (2014, 2015), scaled to match the distance and extinction of iPTF13bvn (see the text). Long-dashed horizontal lines indicate the magnitude of the pre-explosion object in $B V I$ ( $1 \sigma$ uncertainties are indicated with dotted lines). The decline rate from ${ }^{56} \mathrm{Co}$ decay is indicated with a short dotted line. Right panel: SED of the source detected in pre-SN images (red circles) and at $\sim 740$ days (green diamonds and green arrow for the detection limit in the F225W band). Photometry of SN $2011 \mathrm{dh}$ at a similar age scaled to the distance and extinction of iPTF13bvn is shown with black squares. Synthetic photometry from a nebular spectrum of iPTF13bvn scaled to the observed F555W flux is shown with open diamonds. The dashed line shows the $20 M_{\odot}$ O-type star ("Ostar1") of Kim et al. (2015), which represents an accreting companion star at the moment of the SN explosion.

Table 3

KAIT and Nickel Photometry of iPTF13bvn

\begin{tabular}{|c|c|c|c|c|c|c|}
\hline MJD & $\begin{array}{l}\text { Phase }^{\mathrm{a}} \\
\text { (days) }\end{array}$ & $\begin{array}{c}B \\
(\mathrm{mag})\end{array}$ & $\begin{array}{c}V \\
(\mathrm{mag})\end{array}$ & $\begin{array}{c}R \\
(\mathrm{mag})\end{array}$ & $\begin{array}{c}I \\
(\mathrm{mag})\end{array}$ & Telescope \\
\hline 56461.28 & 2.03 & $17.94(14)$ & $17.25(07)$ & $16.92(05)$ & $16.88(09)$ & KAIT \\
\hline 56465.22 & 5.95 & $16.68(12)$ & $16.02(06)$ & $15.76(05)$ & $15.68(08)$ & KAIT \\
\hline 56466.21 & 6.94 & $16.40(11)$ & $15.82(05)$ & $15.64(03)$ & $15.55(04)$ & KAIT \\
\hline 56469.30 & 10.02 & $16.04(10)$ & $15.54(04)$ & $15.20(04)$ & $15.18(06)$ & KAIT \\
\hline 56470.27 & 10.98 & $15.90(06)$ & $15.39(04)$ & $15.20(04)$ & $15.10(04)$ & KAIT \\
\hline
\end{tabular}

Note. Uncertainties in parentheses in units of $0.01 \mathrm{mag}$.

${ }^{a}$ Rest-frame phase after explosion (JD = 2456459.24).

(This table is available in its entirety in machine-readable form.)

We also obtained $B V R I$ imaging of iPTF13bvn until $\sim 280$ days with the Katzman Automatic Imaging Telescope (KAIT; Filippenko et al. 2001) and the $1 \mathrm{~m}$ Nickel telescope at Lick Observatory. Template subtraction was performed using additional images obtained after the $\mathrm{SN}$ faded below detection.
All images were reduced using a custom pipeline (Ganeshalingam et al. 2010). Point-spread-function (PSF) photometry was measured with the DAOPHOT package (Stetson 1987). Apparent magnitudes were first measured in the KAIT4 natural system and then transformed to the standard system using local 
calibrators and color terms as given in Table 4 of Ganeshalingam et al. (2010). We present the resulting light curves in Table 3 and the left panel of Figure 2.

\subsection{Distance and Extinction}

In the current analysis, we adopted a distance to NGC 5806 of $25.8 \pm 2.3 \mathrm{Mpc}$ as provided by the NASA/IPAC Extragalactic Database (NED). This value is similar to that used by Bersten et al. (2014) and about $10 \%(1 \sigma)$ greater than the $22.5 \mathrm{Mpc}$ extensively adopted in the literature on iPTF13bvn.

Milky Way reddening in the direction to iPTF13bvn is $E(B-V)_{\mathrm{MW}}=0.045 \mathrm{mag}$ (Schlafly \& Finkbeiner 2011). Cao et al. (2013) found a total color excess of $E(B-V)_{\text {tot }}=0.07$ mag by measuring the equivalent width of $\mathrm{Na} I \mathrm{D}$ lines in the SN spectrum and using the relation by Poznanski et al. (2012). However, Phillips et al. (2013) pointed out that such a relation underestimates the uncertainty in the extinction. Using observed colors in comparison with a sample of strippedenvelope SNe, Bersten et al. (2014) found a larger value of $E(B-V)_{\text {tot }}=0.21 \pm 0.03$ mag. Srivastav et al. (2014) favored this larger reddening value by comparing the $V-R$ color with the calibration of intrinsic color provided by Drout et al. (2011). We thus adopted the larger reddening value and computed extinction in all bands using the reddening law of Cardelli et al. (1989) with a standard coefficient of $R_{V}=3.1$. Where indicated, we also considered the shorter distance and lower extinction values from Cao et al. (2013).

\section{THE PROGENITOR OF iPTF13bvn}

With the magnitudes and detection limit listed in Table 2, we constructed the spectral energy distribution (SED) of the preand post-SN source, as shown in the right panel of Figure 2. The source at the SN location faded below the pre-explosion level in 2015. The decrease is significant in the F435W/ F438W and F555W bands (by $\sim 3 \sigma$ and $\sim 4 \sigma$, respectively), and marginal in F814W. To test this observation, we performed pre-SN minus post-SN image subtractions, using AstroDrizzle to register and degrade the post-SN images to match the pre-SN images. Then we scaled the flux and subtracted with standard IRAF routines. As shown in Figure 1, the subtractions leave detectable sources at the SN location in F435W/F438W and F555W, and only noise in F814W. We performed aperture photometry of the residual object using DAOPHOT, which led to the magnitudes and upper limit listed in Table 2. It is reassuring that the flux in the subtracted images closely matches the subtraction of fluxes measured in the 2005 and 2015 images.

Assuming that the flux decrease is not caused by large amounts of dust formed in the SN ejecta (see Section 3.1), this result confirms the first association of a SN Ib with its progenitor object. However, deriving a conclusion about the progenitor nature requires some interpretation of the new measurements. In principle, the flux in 2015 could be produced by any combination of the following: the fading $\mathrm{SN}$, a light echo, or an underlying object or population related (or not) to the SN. If what we detected is purely the $\mathrm{SN}$, then the pre-SN source is the progenitor itself. In the opposite extreme, if the $\mathrm{SN}$ makes a negligible contribution, then the progenitor is revealed in the subtraction of pre-SN minus post-SN images (assuming no variability of the environment). Any intermediate situation is theoretically possible. In the following, we will analyze both extreme cases.

\subsection{Case SN: The Fading $S N$}

In order to tell if the detected flux in 2015 was caused by the SN, we compared it with available data on similar events. Unfortunately, there are no observations of other $\mathrm{SNe} \mathrm{Ib}$ at such a late phase. The only published multiband light curves of stripped-envelope $\mathrm{SNe}$ that extend over 700 days are those of the Type IIb SN 1993J and SN 2011dh. However, these SNe may be affected by stronger emission from shock interaction than iPTF13bvn. Indeed, after 500 days SN 1993J showed evidence of prominent interaction emission that flattened the optical light curves (Zhang et al. 2004). In contrast, SN 2011dh appears to have been relatively free of interaction (e.g., see, Maeda et al. 2014; Jerkstrand et al. 2015). To test this we performed a simple, one-zone radioactive deposition calculation (Maeda et al. 2003), assuming full positron trapping and $\gamma$-ray optical depth based on the ejected mass and explosion energy given by Bersten et al. (2012). The resulting optical emission is enough to account for the late-time observations of SN 2011dh without the need to invoke strong interaction.

Figure 2 (left panel) shows the $B V R r$ 'I light curves of $\mathrm{SN}$ 2011dh (Ergon et al. 2014, 2015; Van Dyk et al. 2013), assuming $d=7.8 \mathrm{Mpc}$ and $A_{V}=0.1 \mathrm{mag}$ (Folatelli et al. 2014), and scaled to the distance and extinction of iPTF13bvn. We extrapolated these light curves to 740 days (dotted lines), assuming an average decline rate of $0.007 \pm$ 0.002 mag day $^{-1}$ measured between 600 and 700 days. The extrapolation uncertainty was summed in quadrature with the uncertainty of the latest observed points. We decided not to include later observations of SN 2011dh published by Maund et al. (2015) because they were obtained at $\sim 1160$ days (i.e., $>400$ days after the epoch of our observations), when different emission mechanisms may dominate. The right panel of Figure 2 shows that the resulting SED of SN 2011dh is very similar to that of iPTF13bvn, only slightly fainter in the F435W/F438W band, suggesting that our new images of iPTF13bvn reveal the fading SN.

We also computed spectrophotometry with the spectrum of iPTF13bvn obtained at 306 days by Kuncarayakti et al. (2015). The synthetic fluxes are shown in the right panel of Figure 2, scaled down to reproduce the observed magnitude of $\mathrm{F} 555 \mathrm{~W}=26.72$ at 740 days. The overall SED shape is similar to our measurements at 740 days, which may imply that the spectrum did not evolve significantly. Along with the similarity with SN 2011dh, this suggests the absence of strong interaction or large dust formation, unless both effects canceled each other. The small "excess" in the F438W band seen at 740 days may hint the presence of a hot companion star, as shown in the figure.

Assuming we detected the fading SN, the pre-SN flux could be attributed primarily to the progenitor. The pre-explosion SED has been compared with possible progenitor models in several previous articles, as described in Section 1. Here, we revisit the progenitor nature using our own pre-explosion photometry and our revised distance and reddening (Section 2.1).

The absolute magnitudes of the progenitor object would be $M_{\mathrm{F} 435 \mathrm{~W}}=-6.95 \pm 0.28 \mathrm{mag}, M_{\mathrm{F} 555 \mathrm{~W}}=-6.69 \pm 0.26 \mathrm{mag}$, and $M_{\mathrm{F} 814 \mathrm{~W}}=-6.61 \pm 0.24 \mathrm{mag}$. The uncertainties were derived by summing in quadrature those in apparent 

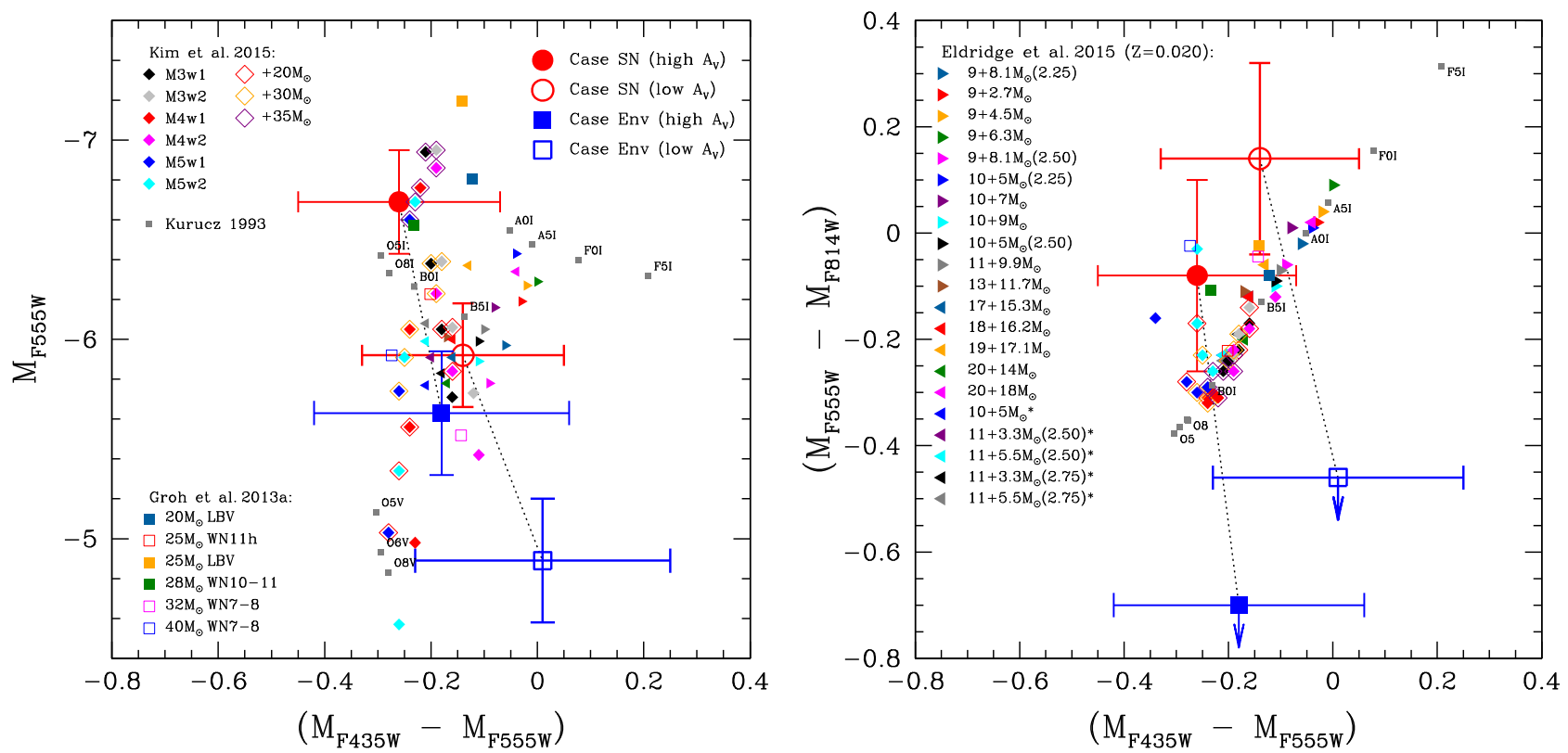

Figure 3. Color-magnitude and color-color diagrams showing the location of the two extreme progenitor alternatives (Case SN and Case Env) discussed in Section 3, compared with previously proposed progenitor models (Groh et al. 2013a; Eldridge et al. 2015; Kim et al. 2015) and stellar atmosphere models (Kurucz 1993). Only solar-metallicity models by E15 are shown, with labels indicating the initial masses of both components. Values in parentheses give the initial orbital separation $\left(\log \left(a / R_{\odot}\right)\right.$, as in Table 1 of E15) for systems of equal masses. Dotted lines join both extreme cases. "High $A_{V}$ " indicates our assumed distance and extinction (Section 2.1), and "low $A_{V}$ " is for values adopted by Cao et al. (2013). Arrows in the right panel indicate upper limits for Case Env.

magnitude, extinction, and distance. Intrinsic colors would be $\left(M_{\mathrm{F} 435 \mathrm{~W}}-M_{\mathrm{F} 555 \mathrm{~W}}\right)=-0.26 \pm 0.19 \quad$ mag $\quad$ and $\left(M_{\mathrm{F} 555 \mathrm{~W}}-M_{\mathrm{F} 814 \mathrm{~W}}\right)=-0.08 \pm 0.18 \mathrm{mag}$.

We compared the corrected photometry with available models of single and binary massive stars, as shown in the color-magnitude and color-color diagrams of Figure 3. The rotating models of $20 M_{\odot}$ and $28 M_{\odot}$ of Groh et al. (2013a; green and blue triangles) are in good agreement with the data. The pre-SN objects predicted by these two models are a luminous blue variable (LBV) and a WR star of type WN1011, respectively. Their respective final masses are $7.1 M_{\odot}$ and $10.8 M_{\odot}$. The mass of the latter object is inconsistent with the analysis of the early-time SN light curve. While the LBV model is marginally compatible with the light-curve analysis, its final structure contains significant amounts of hydrogen and would thus produce a Type IIL or IIb SN. We note, however, that there have been claims of the presence of $\mathrm{H} \alpha$, albeit weak, in the spectrum of iPTF13bvn (Kuncarayakti et al. 2015; Reilly et al. 2016; although see Cao et al. 2013 and Srivastav et al. 2014).

We also compared with massive binary models published by Kim et al. (2015) and E15. Although E15 models with initial primary masses below $13 M_{\odot}$ produce faint companion stars in agreement with the constraint in $\mathrm{F} 225 \mathrm{~W}$, those systems are slightly less luminous or redder than our pre-SN measurements. Only those models by Kim et al. (2015) that included the most massive companion star $\left(35 M_{\odot}\right)$ were able to account for the large optical luminosity. However, these companions would be too bright in F225W to comply with our detection limit. As shown in the right panel of Figure 2, only their least massive $\left(20 M_{\odot}\right)$ companion is allowed by our F225W constraint. We note that such would be the limit of the companion mass at the moment of the SN explosion. Binary evolution models have shown that accreting companions evolve upward in the Hertzprung-Russell diagram, remaining near the zero-age main sequence (ZAMS). However, deriving the initial binary configuration would require detailed evolutionary calculations that would only be justified once we confirm the nature of the source observed in 2015 with further observations.

For comparison, Figure 3 also shows the results with the shorter distance and lower extinction (see Section 2.1). The resulting values, indicated with "low $A_{V}$ " in Figure 3 , are $M_{\mathrm{F} 435 \mathrm{~W}}=-6.06 \pm 0.28 \mathrm{mag}, M_{\mathrm{F} 555 \mathrm{~W}}=-5.92 \pm 0.26 \mathrm{mag}$, and $M_{\mathrm{F} 814 \mathrm{~W}}=-6.06 \pm 0.24 \mathrm{mag}$. The agreement with the models by Groh et al. (2013a) and Kim et al. (2015) is worse than with our preferred distance and extinction. Among the binary systems by E15, those with initial masses of 9 and $8.1 M_{\odot}\left(\right.$ and $\left.\log \left(a / R_{\odot}\right)=2.25\right)$ and 10 and $7 M_{\odot}$ agree within $1 \sigma$ of the low $-A_{V}$ photometry.

\subsection{Case Env: The Environment}

We now consider the case that the SN emission became negligible at 740 days. This requires removing the condition of full trapping of radioactive positrons and neglecting any contributions from possible weak interaction or light echo, or assuming much obscuration by dust. By linearly extrapolating the light curves from 200 days, we find the SN flux would contribute $<1 \%$ of the observed flux in F438W and F555W, and $<6 \%$ in $\mathrm{F} 814 \mathrm{~W}$. This would mean that the new observations revealed the $\mathrm{SN}$ environment. The 740 days SED is roughly compatible with an early-A type supergiant star or a young star cluster. At the distance of NGC 5806, the PSF radius comprises several parsecs; thus, the source may or may not be physically related to the SN progenitor. Our data rule out a bloated red-supergiant companion star as proposed by Hirai \& Yamada (2015); only their hottest companion (model c0.5) would be allowed.

Assuming no variability, the pre-SN minus post-SN images would reveal the progenitor object, the exploding star itself, devoid of any binary companion or associated population. The 
absolute magnitudes would be $M_{\mathrm{F} 435 \mathrm{~W}}=-5.81 \pm 0.27 \mathrm{mag}$, $M_{\mathrm{F} 555 \mathrm{~W}}=-5.63 \pm 0.31 \mathrm{mag}$, and $M_{\mathrm{F} 814 \mathrm{~W}}>-4.93 \mathrm{mag}$. If we consider that up to $6 \%$ of the flux in $\mathrm{F} 814 \mathrm{~W}$ could be due to the $\mathrm{SN}$, the progenitor would have $M_{\mathrm{F} 814 \mathrm{~W}} \gtrsim-5.0 \mathrm{mag}$. We can find some progenitor models that are compatible with the F435W and F555W bands (Figure 3, left panel). However, when including the limit in F814W (right panel), the resulting color of $\left(M_{\mathrm{F} 555 \mathrm{~W}}-M_{\mathrm{F} 814 \mathrm{~W}}\right) \lesssim-0.6 \mathrm{mag}$ excludes all of the comparison models. Even if we adopted the shorter distance and lower extinction (Section 2.1), the color $\left(M_{\mathrm{F} 555 \mathrm{~W}}-M_{\mathrm{F} 814 \mathrm{~W}}\right) \lesssim-0.4$ mag would still be beyond the range of known models, although close to the locus of O-type stars. At the same time, the $M_{\mathrm{F} 435 \mathrm{~W}}-M_{\mathrm{F} 555 \mathrm{~W}}$ color worsens. We conclude that the true nature of the progenitor is likely between the two cases, and closer to Case SN (Section 3.1) than to this other extreme.

\section{CONCLUSIONS}

Our new HST images reveal a decrease in flux relative to the pre-explosion source proposed as the progenitor of iPTF13bvn by Cao et al. (2013). Assuming this decrease is not caused by newly formed dust in the ejecta, it confirms the first progenitor identification of an SN Ib.

With the currently available information, it is not possible to provide a definitive characterization of the progenitor. We present some evidence that the flux in the new images was mostly due to the fading SN. In fact, if we assume a negligible contribution from the $\mathrm{SN}$, the derived progenitor $M_{\mathrm{F} 555 \mathrm{~W}}-M_{\mathrm{F} 814 \mathrm{~W}}$ color would be incompatible with known stellar models.

If what we detected was indeed the SN, then most of the preSN flux would be due to the progenitor. Previously proposed progenitor models, either single or binary, need to be revised to account for these new data. For binary progenitors, our detection limit in F225W constrains any hot companion to be less luminous than a late-O main-sequence star, with $\lesssim 20 M_{\odot}$ at the moment of the SN explosion, assuming it is not heavily obscured by dust. Further observations are required to assess the exact contribution from the $\mathrm{SN}$, and thus to disentangle the progenitor nature.

This research is supported by grants GO-13684, GO-13822, and AR-14295 from STScI, which is operated by AURA, Inc., under NASA contract NAS5-26555. A.V.F.'s group is also grateful for funding through NSF grant AST-1211916, the TABASGO Foundation (KAIT and research support), the Sylvia \& Jim Katzman Foundation, Clark and Sharon Winslow, and the Christopher R. Redlich Fund. This research is supported by the WPI Initiative MEXT (Japan), the Japan Society for the Promotion of Science (JSPS) KAKENHI grants 26800100 (K.M.) 23224004, and 26400222 (K.N.), and by the JSPS Open Partnership Bilateral Joint Research Project between Japan and Chile (K.M.). M.H., G.P., and H.K. acknowledge support from the Millennium Institute of Astrophysics (grant IC120009). H.K. also acknowledges
FONDECYT grant 3140563. N.E.R. is supported by PRININAF 2014. R.J.F. acknowledges support from NSF grant AST-1518052 and the Alfred P. Sloan Foundation. A.A.M. acknowledges support by NASA (Hubble Fellowship grant HST-HF-51325.01, under contract NAS 5-26555). Many UC Berkeley undergraduate students helped obtain Lick/Nickel data. Research at Lick Observatory is partially supported by a generous gift from Google.

\section{REFERENCES}

Benvenuto, O. G., Bersten, M. C., \& Nomoto, K. 2013, ApJ, 762, 74 Bersten, M. C., Benvenuto, O. G., Folatelli, G., et al. 2014, AJ, 148, 68 Bersten, M. C., Benvenuto, O. G., Nomoto, K., et al. 2012, ApJ, 757, 31 Cao, Y., Kasliwal, M. M., Arcavi, I., et al. 2013, ApJL, 775, L7 Cardelli, J. A., Clayton, G. C., \& Mathis, J. S. 1989, ApJ, 345, 245 Dalcanton, J. J., Williams, B. F., Seth, A. C., et al. 2009, ApJS, 183, 67 Dessart, L., Hillier, D. J., Livne, E., et al. 2011, MNRAS, 414, 2985 Dolphin, A. E. 2000, PASP, 112, 1383

Drout, M. R., Soderberg, A. M., Gal-Yam, A., et al. 2011, ApJ, 741, 97

Eldridge, J. J., Fraser, M., Maund, J. R., \& Smartt, S. J. 2015, MNRAS, 446, 2689

Eldridge, J. J., Fraser, M., Smartt, S. J., Maund, J. R., \& Crockett, R. M. 2013, MNRAS, 436, 774

Eldridge, J. J., \& Maund, J. R. 2016, arXiv:1604.05050

Ergon, M., Jerkstrand, A., Sollerman, J., et al. 2015, A\&A, 580, A142

Ergon, M., Sollerman, J., Fraser, M., et al. 2014, A\&A, 562, A17

Filippenko, A. V., Li, W. D., Treffers, R. R., \& Modjaz, M. 2001, in ASP. Conf. Ser. 246, IAU Coll. 183: Small Telescope Astronomy on Global Scales, ed. B. Paczynski, W.-P. Chen, \& C. Lemme (San Francisco, CA: ASP), 121

Folatelli, G., Bersten, M. C., Benvenuto, O. G., et al. 2014, ApJL, 793, L22 Fremling, C., Sollerman, J., Taddia, F., et al. 2014, A\&A, 565, A114 Ganeshalingam, M., Li, W., Filippenko, A. V., et al. 2010, ApJS, 190, 418 Groh, J. H., Georgy, C., \& Ekström, S. 2013, A\&A, 558, L1

Groh, J. H., Meynet, G., Georgy, C., \& Ekström, S. 2013, A\&A, 558, A131

Hachinger, S., Mazzali, P. A., Taubenberger, S., et al. 2012, MNRAS, 422, 70 Harris, W. E. 1990, PASP, 102, 949

Heger, A., Fryer, C. L., Woosley, S. E., Langer, N., \& Hartmann, D. H. 2003, ApJ, 591, 288

Hirai, R., \& Yamada, S. 2015, ApJ, 805, 170

Jerkstrand, A., Ergon, M., Smartt, S. J., et al. 2015, A\&A, 573, A12

Kim, H.-J., Yoon, S.-C., \& Koo, B.-C. 2015, ApJ, 809, 131

Kuncarayakti, H., Maeda, K., Bersten, M. C., et al. 2015, A\&A, 579, A95

Kurucz, R. 1993, ATLAS9 Stellar Atmosphere Programs and 2 km/s grid. Kurucz CD-ROM No. 13 (Cambridge, MA: Smithsonian Astrophysical Observatory)

Liu, Z.-W., Tauris, T. M., Röpke, F. K., et al. 2015, A\&A, 584, A11

Maeda, K., Katsuda, S., Bamba, A., Terada, Y., \& Fukazawa, Y. 2014, ApJ, 785,95

Maeda, K., Mazzali, P. A., Deng, J., et al. 2003, ApJ, 593, 931

Maund, J. R., Arcavi, I., Ergon, M., et al. 2015, MNRAS, 454, 2580

Phillips, M. M., Simon, J. D., Morrell, N., et al. 2013, ApJ, 779, 38

Podsiadlowski, P., Joss, P. C., \& Hsu, J. J. L. 1992, ApJ, 391, 246

Poznanski, D., Prochaska, J. X., \& Bloom, J. S. 2012, MNRAS, 426, 1465

Reilly, E., Maund, J. R., Baade, D., et al. 2016, MNRAS, 457, 288

Schlafly, E. F., \& Finkbeiner, D. P. 2011, ApJ, 737, 103

Shigeyama, T., Nomoto, K., Tsujimoto, T., \& Hashimoto, M.-A. 1990, ApJL, 361, L23

Smith, N., Li, W., Filippenko, A. V., \& Chornock, R. 2011, MNRAS, 412, 1522

Srivastav, S., Anupama, G. C., \& Sahu, D. K. 2014, MNRAS, 445, 1932

Stetson, P. B. 1987, PASP, 99, 191

Van Dyk, S. D., Zheng, W., Clubb, K. I., et al. 2013, ApJL, 772, L32

Zhang, T., Wang, X., Zhou, X., et al. 2004, AJ, 128, 1857 